\title{
Front Matter: Volume 7199
}

, "Front Matter: Volume 7199," Proc. SPIE 7199, Free-Space Laser Communication Technologies XXI, 719901 (11 March 2009); doi: $10.1117 / 12.824383$

SPIE Event: SPIE LASE: Lasers and Applications in Science and Engineering, SPIE. 2009, San Jose, California, United States 


\section{PROCEEDINGS OF SPIE}

\section{Free-Space Laser Communication Technologies $X X I$}

Hamid Hemmati

Editor

28-29 January 2009

San Jose, California, USA

Sponsored and Published by

SPIE 
The papers included in this volume were part of the technical conference cited on the cover and title page. Papers were selected and subject to review by the editors and conference program committee. Some conference presentations may not be available for publication. The papers published in these proceedings reflect the work and thoughts of the authors and are published herein as submitted. The publisher is not responsible for the validity of the information or for any outcomes resulting from reliance thereon.

Please use the following format to cite material from this book:

Author(s), "Title of Paper," in Free-Space Laser Communication Technologies XXI, edited by Hamid Hemmati, Proceedings of SPIE Vol. 7199 (SPIE, Bellingham, WA, 2009) Article CID Number.

ISSN 0277-786X

ISBN 9780819474452

Published by

SPIE

P.O. Box 10, Bellingham, Washington 98227-0010 USA

Telephone +1 3606763290 (Pacific Time) · Fax +1 3606471445

SPIE.org

Copyright (C) 2009, Society of Photo-Optical Instrumentation Engineers

Copying of material in this book for internal or personal use, or for the internal or personal use of specific clients, beyond the fair use provisions granted by the U.S. Copyright Law is authorized by SPIE subject to payment of copying fees. The Transactional Reporting Service base fee for this volume is $\$ 18.00$ per article (or portion thereof), which should be paid directly to the Copyright Clearance Center (CCC), 222 Rosewood Drive, Danvers, MA 01923. Payment may also be made electronically through CCC Online at copyright.com. Other copying for republication, resale, advertising or promotion, or any form of systematic or multiple reproduction of any material in this book is prohibited except with permission in writing from the publisher. The CCC fee code is 0277-786X/09/\$18.00.

Printed in the United States of America.

Publication of record for individual papers is online in the SPIE Digital Library.

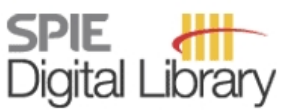

SPIEDigitalLibrary.org

Paper Numbering: Proceedings of SPIE follow an e-First publication model, with papers published first online and then in print and on CD-ROM. Papers are published as they are submitted and meet publication criteria. A unique, consistent, permanent citation identifier (CID) number is assigned to each article at the time of the first publication. Utilization of CIDs allows articles to be fully citable as soon they are published online, and connects the same identifier to all online, print, and electronic versions of the publication. SPIE uses a six-digit CID article numbering system in which:

- The first four digits correspond to the SPIE volume number.

- The last two digits indicate publication order within the volume using a Base 36 numbering system employing both numerals and letters. These two-number sets start with $00,01,02,03,04$, $05,06,07,08,09,0 A, 0 B \ldots 0 Z$, followed by 10-1Z, 20-2Z, etc.

The CID number appears on each page of the manuscript. The complete citation is used on the first page, and an abbreviated version on subsequent pages. Numbers in the index correspond to the last two digits of the six-digit CID number. 


\section{Contents}

vii Conference Committee

\section{SESSION 1 INVITED SESSION I}

719902 Technology needs for next-generation spaceborne lasercom systems (Invited Paper) [7199-01]

R. W. Burch, The Boeing Co. (United States)

719903 Optical data downlinks from Earth observation platforms (Invited Paper) [7199-02]

D. Giggenbach, J. Horwath, M. Knapek, German Aerospace Ctr. (Germany)

719904 Research and development activities on space laser communications in NICT (Invited Paper) [7199-03]

H. Kunimori, Y. Shoji, M. Toyoshima, Y. Takayama, National Institute of Information and Communications Technology (Japan)

\section{SESSION 2 INVITED SESSION II}

$719906 \quad 5.6$ Gbps optical intersatellite communication link (Invited Paper) [7199-05]

B. Smutny, H. Kaempfner, G. Muehlnikel, U. Sterr, B. Wandernoth, F. Heine, U. Hildebrand,

D. Dallmann, M. Reinhardt, A. Freier, R. Lange, K. Boehmer, T. Feldhaus, J. Mueller,

A. Weichert, P. Greulich, S. Seel, Tesat-Spacecom GmbH \& Co. KG (Germany); R. Meyer, Deutsches Zentrum für Luft- und Raumfahrt e.V. (Germany); R. Czichy, Synopta (Switzerland)

\section{SESSION 3 DEMONSTRATIONS}

719907 Experimental evaluation of a radio-on-FSO communication system for multiple RF signal transmission [7199-06]

K. Kazaura, P. Dat, A. Bekkali, A. Shah, T. Suzuki, K. Wakamori, M. Matsumoto, Waseda Univ. (Japan); T. Nakamura, K. Takahashi, T. Higashino, Y. Aburakawa, K. Tsukamoto, S. Komaki, Osaka Univ. (Japan)

719908 Compact free-space optical terminal for multi-gigabit signal transmissions with a single-mode fiber [7199-07]

Y. Arimoto, National Institute of Information and Communications Technology (Japan)

719909 Aircraft to ground unidirectional laser-communications terminal for high-resolution sensors [7199-08]

J. Horwath, C. Fuchs, German Aerospace Ctr. (Germany)

7199 OA Demonstration of a high-efficiency free-space optical communications link [7199-09]

K. Birnbaum, W. Farr, J. Gin, B. Moision, K. Quirk, M. Wright, Jet Propulsion Lab. (United States) 
7199 OB Results from the DOLCE (Deep Space Optical Link Communications Experiment) project [7199-10]

G. Baister, K. Kudielka, T. Dreischer, M. Tüchler, Oerlikon Space AG (Switzerland)

7199 OC Robust short-pulse high-peak-power laser transmitter for optical communications [7199-11] M. W. Wright, Jet Propulsion Lab. (United States)

7199 OD Compensation of large-diameter optical system aberrations with spatial light modulators and deformable mirrors [7199-12]

H. Hemmati, Y. Chen, Jet Propulsion Lab. (United States)

7199 OE Mid-infrared interband cascade lasers for free-space laser communication [7199-13] A. Soibel, M. Wright, W. Farr, S. Keo, C. Hill, Jet Propulsion Lab. (United States); R. Q. Yang, Univ. of Oklahoma (United States); H. C. Liu, National Research Council (Canada)

\section{SESSION 4 ACQUISITION, TRACKING, AND POINTING}

$7199 \mathrm{OH} \quad$ Autonomous access links using laser communications [7199-16]

J. M. Kovalik, A. Biswas, J. R. Charles, M. Regehr, Jet Propulsion Lab. (United States)

7199 ol A sub-hertz vibration isolation plafform for a deep space optical communication transceiver [7199-17]

V. Sannibale, G. G. Ortiz, W. H. Farr, Jet Propulsion Lab. (United States)

\section{SESSION 5 SYSTEMS UNDER DEVELOPMENT}

7199 OK Canonical deep space optical communications transceiver [7199-19]

G. G. Ortiz, W. H. Farr, J. R. Charles, W. T. Roberts, V. Sannibale, J. Gin, A. Saharaspude,

V. Garkanian, Jet Propulsion Lab. (United States)

7199 OL Compact deep-space optical communications transceiver [7199-20]

W. T. Roberts, J. R. Charles, Jet Propulsion Lab. (United States)

7199 OM BER performance of MIMO diffuse free-space optical systems [7199-21]

D. S. Pfeil, S. Vamsidhar, T. P. Kurzweg, K. R. Dandekar, Drexel Univ. (United States)

7199 ON Combined laser communications and laser ranging transponder for Moon and Mars [7199-22]

H. Hemmati, K. M. Birnbaum, W. H. Farr, S. Turyshev, A. Biswas, Jet Propulsion Lab. (United States)

719900 A new deformable mirror and experimental setup for free-space optical communication [7199-32]

F. Rooms, S. Camet, J. Charton, J.-F. Curis, L. Jocou, ALPAO (France)

7199 OP Modified PN code laser modulation technique for laser measurements [7199-33]

X. Sun, J. B. Abshire, NASA Goddard Space Flight Ctr. (United States) 


\section{SESSION 6 DETECTORS AND RECEIVERS}

$71990 Q \quad$ Negative avalanche feedback detectors for photon-counting optical communications [7199-23]

W. H. Farr, Jet Propulsion Lab. (United States)

\section{SESSION 7 ANALYSIS}

7199 OS Virtual array receiver options for 64-ary pulse position modulation (PPM) [7199-26]

A. J. Mendez, Mendez R\&D Associates (United States); V. J. Hernandez, Lawrence Livermore National Lab. (United States); R. M. Gagliardi, Univ. of Southern California (United States); C. V. Bennett, Lawrence Livermore National Lab. (United States)

7199 OT Improved bit error rate estimation over experimental optical wireless channels [7199-27] M. El Tabach, France Télécom (France); S. Saoudi, TELECOM Bretagne, CNRS Lab-STICC, Univ. européenne de Bretagne (France); P. Tortelier, O. Bouchet, France Télécom (France); R. Pyndiah, TELECOM Bretagne, CNRS Lab-STICC, Univ. européenne de Bretagne (France)

7199 OV Using MIMO transmissions in free-space optical communications in presence of clouds and turbulence [7199-29]

Z. Hajjarian, M. Kavehrad, The Pennsylvania State Univ. (United States)

Author Index 
Downloaded From: https://www.spiedigitallibrary.org/conference-proceedings-of-spie on 26 Apr 2023

Terms of Use: https://www.spiedigitallibrary.org/terms-of-use 


\title{
Conference Committee
}

\author{
Symposium Chairs \\ Donald J. Harter, IMRA America, Inc. (United States) \\ Peter R. Herman, University of Toronto (Canada) \\ Symposium Cochairs
}

Henry Helvajian, The Aerospace Corporation (United States)

Friedrich G. Bachmann, Rofin-Sinar Laser GmbH (Germany)

Conference Chair

Hamid Hemmati, Jet Propulsion Laboratory (United States)

Program Committee

Guy C. Baister, Oerlikon Space AG (Switzerland)

David Begley, Ball Aerospace \& Technologies Corporation (United States)

Don M. Boroson, MIT Lincoln Laboratory (United States)

Robert T. Carlson, BAE Systems (United States)

Wayne R. Fenner, The Aerospace Corporation (United States)

Yoshisada Koyama, National Institute of Information and Communications Technology (Japan)

Robert Lange, Tesat-Spacecom GmbH \& Co. KG (Germany)

Donald J. Nicholson, Air Force Research Laboratory (United States)

Zoran Sodnik, European Space Agency (Netherlands)

Morio Toyoshima, National Institute of Information and

Communications Technology (Japan)

Alan E. Willner, University of Southern California (United States)

Shiro Yamakawa, Japan Aerospace Exploration Agency (Japan)

Session Chairs

1 Invited Session I

Hamid Hemmati, Jet Propulsion Laboratory (United States)

2 Invited Session II

Hamid Hemmati, Jet Propulsion Laboratory (United States)

3 Demonstrations

Don M. Boroson, MIT Lincoln Laboratory (United States) 
$4 \quad$ Acquisition, Tracking, and Pointing

Ronald W. Burch, The Boeing Co. (United States)

5 Systems Under Development

William H. Farr, Jet Propulsion Laboratory (United States)

6 Detectors and Receivers

Hamid Hemmati, Jet Propulsion Laboratory (United States)

7 Analysis

Abhijit Biswas, Jet Propulsion Laboratory (United States) 